\section{G543(P) IMPROVING PATIENT SAFETY AND QUALITY OF CARE FOR REGIONAL NEONATAL SERVICES}

N Hoque, S Wang, M Watson. Curriculum Delivery Group, London School of Paediatrics, London, UK

\subsection{6/archdischild-2015-308599.494}

Context Neonatal care delivered by ST1 Paediatric trainees attached to 18 Local Neonatal Units and Special Care Baby Units.

Problem Trainees did not feel adequately prepared or supported to perform essential duties of their role including attending deliveries and performing newborn examinations. Trainees highlighted the risks to patient safety and quality of care.

Assessment of problem and analysis of its causes To quantify the problem and understand the root causes we interviewed and surveyed 48 Paediatric trainees after completing their first post with neonatal duties. $50 \%$ of trainees felt inadequately prepared to perform essential duties and $40 \%$ of trainees were unsatisfied with their supervision. We discussed our findings with trainees, the specialty training committee and local departments, and identified problems with induction and training as well as devising strategic interventions.

Intervention Multiple interventions included 1. Neonatal Life Support (NLS) courses specifically for ST1 trainees before or during their first neonatal post and 2. Engaging with College Tutors to provide comprehensive recommendations for neonatal specific induction, adequate supervision attending deliveries and performing newborn checks, and formal assessment of competence before working unsupervised.

Study design Trainees who had completed their first neonatal post were surveyed. We used multiple choice, Likert scale and free text answers to capture trainee's experiences.

Strategy for change We implemented changes to training over two years, starting with NLS training for new ST1s. Working with Training Program Directors and NLS course directors we offered places to all new ST1s at or near induction. With Local Education Providers and neonatologists, and guided by trainee' feedback, we developed standards and recommendations for local ST1 induction. To aid dissemination, at all stages, we presented the work at regional and local meetings and directly communicated with the Specialty Training Committee, College Tutors, and trainees.

Measurement of improvement We found that undertaking NLS training before starting their first post significantly increased trainees' confidence in attending deliveries and resuscitating newborns. Trainees felt better prepared to perform their duties if they had a specific neonatal induction lasting at least two days (our recommendation). Trainees satisfaction with supervision at deliveries was significantly increased when they were accompanied to the first 4 or more deliveries (our recommendation). There was a trend towards increased satisfaction with supervision when trainees were observed for the first 3 or more newborn checks.

Effects of changes Our work increased the proportion of ST1s a. adequately trained to resuscitate newborns before starting work, b. adequately supervised after induction, and c. having an assessment of competence before working alone utilising Supervised Learning Events. We decreased the number of inadequately trained staff who are responsible for neonatal care and improved supervision that ultimately increased patient safety. Our ongoing challenge is supporting local units to balance service provision with effective training and supervision.
Lessons learnt We learnt that effective change relied upon good communication at all levels between lead providers, local units, and trainees. We focused on Paediatric trainees but other trainees perform the same duties in some units including GPVTS and FY2 trainees. It is important to understand their experience and support them in similar ways in order to protect patient safety.

Message for others Safe neonatal care relies on adequately trained and supervised junior medical staff. Significant shortcomings were partly addressed using a collaborative approach including regional courses and support of local training. We believe that quality of care was improved by increasing the proportion of trained staff that are assessed before working independently.

\section{G544(P) ABSTRACT WITHDRAWN}

\section{G545(P) CLINICAL MANAGEMENT AND DEVELOPING SERVICE IMPROVEMENT PROJECTS (SIP) - OUR EXPERIENCE}

S Jyothi, A Kanani, H Goodyear. Paediatrics, West Midlands, Birmingham, UK

\subsection{6/archdischild-2015-308599.495}

Context Paediatric trainees have variable training in clinical management. Although all trainees are involved in audits and many in research, not many trainees understand how NHS management works.

Assessment of problem and analysis of its causes An informal discussion among senior trainees revealed that almost all were planning to attend a leadership or management course during their last year of training and specifically before attending consultant interviews. Most of these courses were oversubscribed and had trainees from different specialties leading to difficulty in following examples of change in clinical practice.

Intervention A senior trainee (ST7) liaised with the Head of School who agreed to fund this initiative for 2 years. We made enquires to other deaneries and 3 universities who organised medical management courses. We chose Keele University to collaborate with for a new bespoke course for senior paediatric trainees. The deanery allocated $£ 30,000$ for 60 trainees to complete this course over a 2 year period. Each course was limited to 22 trainees to encourage discussion and interaction.

Strategy for change Guided by the senior trainee who initiated this project, a 4-day programme was developed on Clinical Management for paediatric trainees. Course information was disseminated via email to all senior trainees and announced in ST6-8 training days The first course was run in autumn 2012. The programme included an initial 2 days, an 8-10 week interval to complete a SIP before the final 2 days.

Main course topics

- History and structure of NHS

- Financing healthcare

- Personality types

- Leading and motivating teams

- Leadership style and handling conflict

- Roles and responsibilities as a new consultant

- Writing a successful business case

- Service improvement project-the basics 
Measurement of improvement Feedback from 2 cohorts (42 trainees) was consistently positive with all trainees gaining insight into topics which are not usually covered during training. Having a bespoke course helped speakers to discuss examples of paediatric issues and their management. Limiting the number of trainees helped to promote discussion as intended.

Effects of changes By 2014, all level 3 trainees in our deanery had completed this course. Trainees completed a SIP and presented it to their group on the final day of the course. Trainees were encouraged to discuss their projects and offered support with the SIPs.

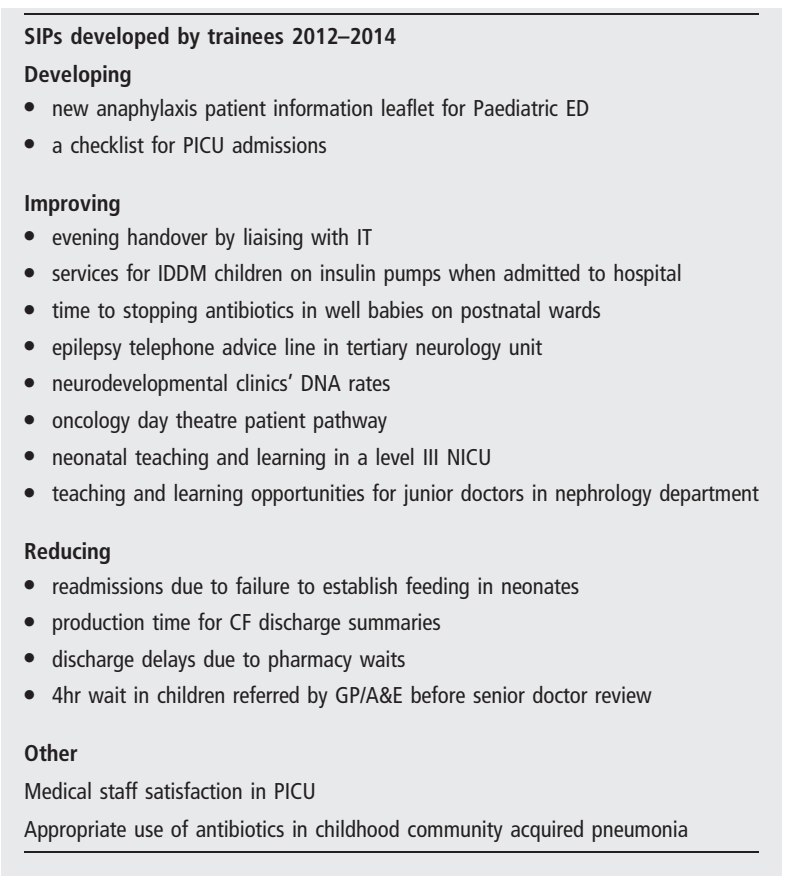

After review of feedback, we decided to run this course yearly for the next 5 years. The deanery has encouraged trainees to improve their understanding of clinical management by providing leave and funding. This has also helped individual hospital trusts by improving clinical care due to trainees SIPs.

Message for others We feel that early exposure to knowledge of clinical management will encourage trainees to help lead change in the future.

\section{G546(P) THE ASSESSMENT AND MANAGEMENT OF THE FEBRILE CHILD UNDER FIVE IN THE PRIMARY CARE SETTING}

A Andrews. Paediatrics, Royal Alexandra Children's Hospital, Brighton, UK

\subsection{6/archdischild-2015-308599.496}

Context This project was performed in a primary setting general practice surgery between October 2013 and January 2014. It involved analysis of data submitted by staff at the surgery including foundation doctors, registrar trainees, general practitioners and nurses.

Problem The aim of the project was to analyse the assessment and management of the febrile child under five years in a primary setting. This topic was chosen as infections was the main reason for children under five presenting to a general practice setting as well as being the leading cause of death. The National Institute for Health and Care Excellence (NICE) fever pathway was updated in May 2013 which provided a good framework for managing these cases and adherence to the fever pathway was assessed.

Assessment of problem and analysis of its causes According to the NICE fever pathway all children presenting with a fever should have their temperature, hear rate, respiratory rate and capillary refill time measured and recorded. Data was collected on the Egton Medical Information System (EMIS) retrospectively between May 2013 and August 2013 to determine whether this standard was adhered to. The results showed that only $10 \%$ of patients presenting with a fever has all parameters recorded.

Intervention In order to improve the documentation and assessment of the febrile child this data was presented to members of the practice in a team meeting highlighting the importance of measuring all the parameters in assessment of a child with a fever. A template was also designed to make this documentation more effective and presented to staff at the meeting. This template was added to EMIS and would be highlighted once the terms fever or temperature is documented on EMIS for any child under the age of 5 .

Study design The project was designed as an audit using the NICE fever pathway as the standard.

Strategy for change A teaching session was set up including all practice members. The data was presented as well as the recently improved NICE guidelines and traffic light system. Feedback from staff members on ways to improve data recording was encouraged through an informal discussion. The febrile child template was also presented to staff members showing them how it will be accessed and explaining how it should be used with reference to the NICE standards.

Measurement of improvement The impact of the intervention was determined by reassessing the data on EMIS after installing the febrile child template. Information entered on EMIS between November 2013 to January 2014 for each child presenting with a fever was analysed to determine if the assessment adhered to NICE standards.

Effects of changes The re-audit showed that following the teaching session on the assessment of the febrile child and the implementation of a template for recording the data there was a statistically significant improvement in assessing the febrile child with $62 \%$ having all parameters recorded in comparison to $10 \%$ on initial audit. This showed an improvement in management of these cases through accurate referrals to secondary care as well as appropriate use of antibiotics and safety net thus showing an overall improvement in clinical care.

Lessons learnt This project highlighted that simple interventions such as teaching sessions and the use of templates for recording data can improve clinical care of patients.

Message for others In busy clinical settings proformas and templates are helpful ways to standardise assessments and management of patients to ensure excellent standards of clinical care.

\section{G547(P) 'JUST CHECKING' - IMPLEMENTING A CHECKLIST}

1,2LC Budd, ${ }^{1,2} \mathrm{~L}$ Bignell, ${ }^{1,2} \mathrm{C}$ Bevan. ${ }^{1}$ Children's Emergency Department, Royal Alexandra Children's Hospital, Brighton, UK; ${ }^{2}$ Department of Paediatrics, Brighton and Sussex University Hospitals NHS Trust, Brighton, UK

\subsection{6/archdischild-2015-308599.497}

Context This quality improvement project as performed in a large District General Hospital, Children's Emergency Department (CED). It reviewed the 'Wheeze Pathway' used to treat children presenting with acute exacerbations of wheeze. These 